\title{
Inflammatory indexes are not associated with sarcopenia in Chinese community- dwelling older people: a cross-sectional study
}

Tianjiao Tang ${ }^{1}$, Lingling Xie ${ }^{1}$, Lingling Tan ${ }^{1}$, Xiaoyi Hu${ }^{1}$ and Ming Yang ${ }^{1,2,3^{*}}$ (D)

\begin{abstract}
Background: Inflammatory indexes (platelet-to-lymphocyte ratio [PLR], neutrophil-to-lymphocyte ratio [NLR], and lymphocyte-to-monocyte ratio [LMR]) are recently supposed to be the biomarkers of sarcopenia. We aimed to validate the association between these inflammatory indexes and sarcopenia in Chinese community-dwelling older people.

Methods: We consecutively recruited community-dwelling older adults aged 60 years or older. The neutrophil, lymphocyte, monocyte, and platelet counts, and C-reactive protein (CRP) were tested using standard methods. Sarcopenia was defined according to different criteria: the Asian Working Group for Sarcopenia (AWGS), the updated version of AWGS (AWGS 2019), the European Working Group on Sarcopenia in Older People (EWGSOP), the updated version of EWGSOP (EWGSOP2), the International Working Group on Sarcopenia (IWGS), and the Foundation for the National Institutes of Health Sarcopenia Project (FNIH). Multiple logistic regression analysis was performed.

Results: We included 384 participants. A total of 61 participants (15.9\%) were diagnosed with sarcopenia according to the AWGS criteria. There was no significant difference in PLR, NLR, LMR, and CRP between the sarcopenia group and the non-sarcopenia group regardless of the diagnostic criteria. No significant association between PLR, NLR, LMR, and AWGS-defined sarcopenia was found (PLR per 1- standard deviation [SD]: adjusted odds ratio [OR] 1.09, 95\% confidence interval [CI] 0.82 to 1.45; NLR per 1-SD: adjusted OR 0.96, 95\% Cl 0.71 to 1.30; LMR per 1-SD: adjusted $\mathrm{OR} 1.01,95 \% \mathrm{Cl} 0.74$ to 1.38). Similar results were found when sarcopenia was defined by different criteria and when PLR, NLR, LMR were treated as categorical variables.

Conclusions: Our study did not support the utility of the inflammatory indexes (NLR, PLR, and LMR) as the biomarkers of sarcopenia in Chinese community-dwelling older people. However, considering the inflammatory indexes can be simply calculated from a routine blood test, further studies in different populations remain warranted.
\end{abstract}

Keywords: Inflammatory indexes, Sarcopenia, Older adults, Muscle wasting, Muscle atrophy

\footnotetext{
* Correspondence: yangmier@gmail.com

${ }^{1}$ Center of Gerontology and Geriatrics, West China Hospital, Sichuan

University, No.37 Guoxue Lane, Chengdu, Sichuan, China

${ }^{2}$ Precision Medicine Research Center, West China Hospital, Sichuan University,

No. 37 Guoxue Lane, Chengdu, Sichuan, China

Full list of author information is available at the end of the article
}

(c) The Author(s). 2020 Open Access This article is licensed under a Creative Commons Attribution 4.0 International License, which permits use, sharing, adaptation, distribution and reproduction in any medium or format, as long as you give appropriate credit to the original author(s) and the source, provide a link to the Creative Commons licence, and indicate if changes were made. The images or other third party material in this article are included in the article's Creative Commons licence, unless indicated otherwise in a credit line to the material. If material is not included in the article's Creative Commons licence and your intended use is not permitted by statutory regulation or exceeds the permitted use, you will need to obtain permission directly from the copyright holder. To view a copy of this licence, visit http://creativecommons.org/licenses/by/4.0/ The Creative Commons Public Domain Dedication waiver (http://creativecommons.org/publicdomain/zero/1.0/) applies to the data made available in this article, unless otherwise stated in a credit line to the data. 


\section{Background}

Sarcopenia was traditionally considered as a geriatric syndrome featured by loss of muscle mass and low muscle strength and/or low physical performance [1]. Recently, it has been formally recognized as a muscle disease with an international classification of disease, tenth revision, clinical modification (ICD-10-CM) Diagnosis Code [2, 3]. Sarcopenia is common among older people and is related to numerous adverse health outcomes, such as falls, functional decline, hospitalization, poor quality of life, and death $[4,5]$. It also increases health economic burden and induces challenges for public health.

Although the pathogenesis of sarcopenia remains unclear, recent evidence shows that chronic low-grade inflammation may play an important role in the development of sarcopenia. For example, inflammation may harm skeletal muscle mass through direct catabolic effects or by causing reduced dietary nutrition intake (induction of anorexia) $[6,7]$.

Many inflammatory markers, such as interleukin- 6 (IL-6), C-reactive protein (CRP), and tumor necrosis factor $\alpha$ (TNF- $\alpha)$, have been linked with sarcopenia [6, $8]$. However, these biomarkers are not routinely tested in community settings when patients have no clinical evidence of inflammation, therefore, this may limit their utility in clinical practice. The inflammatory indexes, i.e., platelet-to-lymphocyte ratio (PLR), neutrophil-tolymphocyte ratio (NLR), and lymphocyte-to-monocyte ratio (LMR), are calculated based on the neutrophil, lymphocyte, monocyte, and platelet counts that are routinely tested in most clinical settings worldwide. They are easily measurable and inexpensive, and have been regarded as important indicators of systemic inflammation $[9,10]$ and serve as predictive biomarkers in patients with coronary heart disease [11], various malignancies [12], and neurodegenerative disease [13]. Recently, a case-control study showed that NLR was an independent predictor for sarcopenia in Turkish older people admitted to the outpatient clinics [14]. Additionally, PLR was reported to be a novel biomarker of sarcopenia in American older people [15]. These findings are valuable but need to be validated in different populations. We, therefore, reanalyzed the data of a crosssectional study to investigate the possible relationship between these inflammatory indexes and sarcopenia in Chinese community-dwelling older people.

\section{Methods}

\section{Study design and participants}

We reanalyzed the data of a cross-sectional study conducted in Chengdu, China, between October to November 2017. The details of the study design were previously reported [16]. Briefly, community-dwelling older people aged 60 years or older were consecutively recruited. The exclusion criteria included individuals with clinical evidence of acute or chronic inflammation, severe mental illness, implanted pacemaker, clinically visible edema, unable to walk, severe renal failure, severe heart failure, and inability to communicate with interviewers. Face-toface interviews were conducted to collect data from all participants by trained interviewers, and anthropometric measurements were performed by trained nurses. All participants or their legal proxies signed the informed consent, and the Research Ethics Committee of Sichuan University approved the study.

\section{Measurement of laboratory parameters}

Venous blood samples were collected in the morning after an overnight fast to measure the laboratory parameters including platelet counts, neutrophil counts, lymphocyte counts, monocyte counts, hemoglobin, fasting plasma glucose, plasma lipids, total bilirubin, direct bilirubin, total protein, albumin, alanine aminotransferase (ALT), aspartate aminotransferase (AST), $\gamma$-glutamyl transpeptidase (GGT), alkaline phosphatase, Cystatin C, creatinine, and uric acid using standard kits (Roche Diagnostics, Switzerland). The level of globulin was obtained indirectly by subtracting the level of albumin from the level of total protein. Additionally, CRP levels were detected with the Modular Analytics Cobas ${ }^{\bullet} 6000$ (Roche Diagnostics, Switzerland) by an immunoturbidimetric technique.

\section{Calculation of the inflammatory indexes}

PLR and NLR were calculated as the absolute platelet counts and neutrophil counts divided by the absolute lymphocyte counts, respectively. LMR was calculated as the absolute lymphocyte counts divided by the absolute monocyte counts.

\section{Measurement of muscle mass, muscle strength, and physical performance}

According to the recommendation of the Asia Working Group for Sarcopenia (AWGS) [17], a bioimpedance analysis (BIA) device (InBody 230; Biospace Co. Ltd., Seoul, Korea) was used to estimate the appendicular skeletal muscle mass (ASM) and body fat mass. Then, the appendicular skeletal muscle mass index (ASMI) was calculated by the equation: ASMI $\left(\mathrm{kg} / \mathrm{m}^{2}\right)=\mathrm{ASM} /$ height ${ }^{2}$. Handgrip strength was measured using a handheld dynamometer (EH101, Xiangshan Inc., Guangdong, China) [17]. After three measurements of the handgrip strength of both hands, the best result of either hand was used for the analyses. Gait speed was measured as the time consumed to walk a 4-m course at usual speed to estimate physical performance $[17,18]$. Canes or 
walkers were allowed during the walking test, if necessary.

\section{Assessment of sarcopenia}

We defined sarcopenia according to different criteria: the AWGS [17], the updated version of AWGS (AWGS 2019) [19], the European Working Group on Sarcopenia in Older People (EWGSOP) [20], the updated version of EWGSOP (EWGSOP2) [2], the International Working Group on Sarcopenia (IWGS) [21], and the Foundation for the National Institutes of Health Sarcopenia Project (FNIH) [22]. The detailed criteria used in this study are listed in Supplementary Table 1.

\section{Assessment of potential covariates}

Covariates such as gender, age, and comorbidities (hypertension, coronary heart disease, diabetes, stroke, chronic obstructive pulmonary disease, cognitive impairment, and history of falls in the previous year) were collected from the face-to-face interviews. The participants' body weight and height were measured by trained nurses. The body mass index (BMI) was then calculated by the equation: BMI $\left(\mathrm{kg} / \mathrm{m}^{2}\right)=$ body weight $/$ height ${ }^{2}$. The calf circumference (CC) was measured around the left calf at the widest part, with the participants lie in a supine position and left knee bent at a right angle, by a millimeter graded tape.

\section{Statistical analysis}

For categorical variables, the data were presented as numbers (percentages), and the chi-squared tests were used to compare the differences between groups. The continuous data were of normal distribution according to the Shapiro-Wilk test. Therefore, for the continuous variables, the data were presented as mean \pm standard deviation (SD), and the differences between groups were compared using the one-way ANOVA test.

Pearson's $\mathrm{r}$ was calculated to detect the correlations between the inflammatory indexes and the component of sarcopenia (ASM, gait speed, and handgrip strength) and CRP. Moreover, multiple logistic regression analysis was performed to calculate the adjusted odds ratios (ORs) and 95\% confidence intervals (CIs) for sarcopenia by the inflammatory indexes and CRP. The inflammatory indexes and CRP were treated both as continuous variables (per 1-SD) and as categorical variables (using quartile cutoff points). Model 1 was adjusted for age and gender. Model 2 further adjusted for coronary heart disease and cognitive impairment. Model 3 included Model $2+$ albumin, high-density lipoprotein cholesterol (HDL$\mathrm{C})$, and BMI.

Data analyses were performed using SPSS version 20.0 (SPSS Inc., Chicago, IL, USA). $P<0.05$ was considered statistically significant.

\section{Results}

Baseline characteristics of the study population

We included 384 participants (224 women and 160 men) with a mean age of $71.5 \pm 5.8$ years. Table 1 shows the characteristics of our study population according to AWGS-defined sarcopenia. Participants with sarcopenia were significantly older than those without sarcopenia $(p<0.001)$. Compared with the non-sarcopenia group, the sarcopenia group was more likely to have coronary heart disease $(18.0 \%$ vs. $7.7 \%, p=0.011)$, cognitive impairment $(13.1 \%$ vs. $1.9 \%, \mathrm{p}<0.001)$, and a history of falls in the previous year $(26.2 \%$ vs. $13.3 \%, p=0.010)$. The level of globulin and HDL-C in the sarcopenia group were significantly higher than those in the nonsarcopenia group, whereas the level of albumin and hemoglobin were significantly lower in the sarcopenia group. Very similar results were found when the study population was grouped according to AWGS 2019defined sarcopenia (Supplementary Table 2).

There were no significant differences concerning the PLR, NLR, and LMR between the sarcopenia group and the non-sarcopenia group regardless of the diagnostic criteria of sarcopenia (Table 2).

\section{Prevalence of sarcopenia}

Among the 384 participants, between 38 and 176 subjects were diagnosed with sarcopenia depending on the diagnostic criteria applied. The prevalence of sarcopenia thus varied from 9.9\% (EWGSOP2) to 45.8\% (AWGS 2019). According to the AWGS criteria, 61 participants (15.9\%) had sarcopenia. The prevalence of sarcopenia was not significantly different in men and women $(11.9 \%$ vs. $18.8 \%$, respectively; $P=0.069$ ).

\section{Correlation between the inflammatory indexes and ASM, gait speed, handgrip strength, and CRP}

As shown in Table 3, PLR was significantly but slightly correlated with ASM $(\mathrm{r}=-0.103, p=0.044)$, while LMR was significantly but slightly correlated with handgrip strength $(r=-0.121, p=0.018)$.

\section{Association between the inflammatory indexes, CRP, and sarcopenia}

As shown in Table 4, PLR, NLR, LMR, or CRP was not significantly associated with AWGS-defined sarcopenia (PLR per 1-SD: OR 1.19, 95\% CI 0.92 to 1.53; NLR per 1-SD: OR $0.97,95 \%$ CI 0.73 to 1.28 ; LMR per 1 -SD: OR $0.99,95 \%$ CI 0.75 to 1.30 ; CRP per $1-S D$ : OR $1.11,95 \%$ CI 0.86 to 1.44). After fully adjusting for multiple covariates, similar results were identified (PLR per 1-SD: adjusted OR $1.09,95 \%$ CI 0.82 to 1.45 ; NLR per 1-SD: adjusted OR $0.96,95 \%$ CI 0.71 to 1.30 ; LMR per 1-SD: adjusted OR 1.01, 95\% CI 0.74 to 1.38 ; CRP per 1-SD: adjusted OR 1.14, 95\% CI 0.85 to 1.54 ). 
Table 1 Baseline characteristics of participants according to AWGS-defined sarcopenia

\begin{tabular}{|c|c|c|c|}
\hline Characteristics & $\begin{array}{l}\text { No sarcopenia } \\
(n=323)\end{array}$ & $\begin{array}{l}\text { Sarcopenia } \\
(n=61)\end{array}$ & $p$ \\
\hline Women (\%) & $182(56.3)$ & $42(68.9)$ & 0.069 \\
\hline Age (years) & $70.7 \pm 5.3$ & $75.9 \pm 6.5$ & $<0.001$ \\
\hline \multicolumn{4}{|l|}{ Comorbidities (\%) } \\
\hline Hypertension & $96(29.7)$ & $20(32.8)$ & 0.632 \\
\hline Coronary heart disease & $25(7.7)$ & $11(18.0)$ & 0.011 \\
\hline Diabetes & $29(9.0)$ & $7(11.5)$ & 0.539 \\
\hline Stoke & $41(12.7)$ & $6(9.8)$ & 0.532 \\
\hline COPD & $28(8.7)$ & $4(6.6)$ & 0.584 \\
\hline Cognitive impairment & $6(1.9)$ & $8(13.1)$ & $<0.001$ \\
\hline History of falls & $43(13.3)$ & $16(26.2)$ & 0.010 \\
\hline BMI $\left(\mathrm{kg} / \mathrm{m}^{2}\right)$ & $24.4 \pm 3.4$ & $23.4 \pm 2.9$ & 0.025 \\
\hline CC $(\mathrm{cm})$ & $32.7 \pm 2.6$ & $30.8 \pm 2.2$ & $<0.001$ \\
\hline ASM (kg) & $15.4 \pm 3.8$ & $12.3 \pm 2.6$ & $<0.001$ \\
\hline ASMI $\left(\mathrm{kg} / \mathrm{m}^{2}\right)$ & $6.3 \pm 1.0$ & $5.4 \pm 0.8$ & $<0.001$ \\
\hline Body fat mass (kg) & $19.0 \pm 5.8$ & $18.5 \pm 5.1$ & 0.531 \\
\hline Gait speed (m/s) & $0.9 \pm 0.2$ & $0.6 \pm 0.1$ & $<0.001$ \\
\hline Handgrip strength (kg) & $24.4 \pm 8.7$ & $14.8 \pm 4.8$ & $<0.001$ \\
\hline \multicolumn{4}{|l|}{ Laboratory parameters } \\
\hline Total bilirubin ( $\mu \mathrm{mol} / \mathrm{L})$ & $15.2 \pm 6.4$ & $15.2 \pm 6.3$ & 0.956 \\
\hline Direct bilirubin $(\mu \mathrm{mol} / \mathrm{L})$ & $5.1 \pm 1.8$ & $4.9 \pm 1.7$ & 0.388 \\
\hline ALT (IU/L) & $19.3 \pm 10.2$ & $20.0 \pm 17.3$ & 0.623 \\
\hline AST (IU/L) & $23.0 \pm 7.0$ & $24.9 \pm 13.4$ & 0.115 \\
\hline Albumin $(g / L)$ & $43.1 \pm 2.6$ & $42.2 \pm 2.9$ & 0.007 \\
\hline Globulin (g/L) & $28.6 \pm 4.2$ & $30.2 \pm 4.7$ & 0.007 \\
\hline Alkaline phosphatase (IU/L) & $78.7 \pm 21.0$ & $81.9 \pm 23.7$ & 0.285 \\
\hline GGT (IU/L) & $23.5 \pm 15.9$ & $24.3 \pm 21.6$ & 0.714 \\
\hline Creatinine $(\mu \mathrm{mol} / \mathrm{L})$ & $74.3 \pm 17.8$ & $73.4 \pm 20.8$ & 0.732 \\
\hline Cystatin C (mg/L) & $1.0 \pm 0.2$ & $1.1 \pm 0.2$ & 0.230 \\
\hline Uric acid $(\mu \mathrm{mol} / \mathrm{L})$ & $332.5 \pm 82.7$ & $311.0 \pm 81.6$ & 0.063 \\
\hline Glucose (mmol/L) & $5.5 \pm 1.4$ & $5.6 \pm 1.8$ & 0.442 \\
\hline Triglyceride (mmol/L) & $1.5 \pm 1.0$ & $1.3 \pm 0.7$ & 0.226 \\
\hline Total cholesterol (mmol/L) & $4.5 \pm 0.9$ & $4.6 \pm 1.0$ & 0.481 \\
\hline $\mathrm{HDL}-\mathrm{C}(\mathrm{mmol} / \mathrm{L})$ & $1.4 \pm 0.4$ & $1.6 \pm 0.4$ & 0.004 \\
\hline LDL-C (mmol/L) & $2.9 \pm 0.8$ & $2.9 \pm 0.9$ & 0.955 \\
\hline CRP (mg/L) & $2.7 \pm 1.9$ & $2.9 \pm 2.0$ & 0.420 \\
\hline Hemoglobin (g/L) & $137.2 \pm 15.6$ & $129.2 \pm 16.1$ & $<0.001$ \\
\hline Platelet $(10 \wedge 9 / L)$ & $148.8 \pm 52.3$ & $157.6 \pm 59.7$ & 0.240 \\
\hline Leukocyte $(10 \wedge 9 / L)$ & $5.5 \pm 1.4$ & $5.4 \pm 1.6$ & 0.488 \\
\hline Neutrophil $(10 \wedge 9 / L)$ & $3.3 \pm 1.0$ & $3.2 \pm 1.2$ & 0.638 \\
\hline Lymphocyte (10^9/L) & $1.7 \pm 0.5$ & $1.6 \pm 0.5$ & 0.482 \\
\hline Monocyte (10^9/L) & $0.4 \pm 0.2$ & $0.4 \pm 0.1$ & 0.406 \\
\hline
\end{tabular}

Notes: Data are presented as the number (percentage) for the following variables: women and specific comorbidities listed above. For other variables, the mean \pm SD is applied

One-way ANOVA and chi-squared tests were used where appropriate. $\mathrm{P}<0.05$ indicates statistically significant

Abbreviations: $A L T$ alanine aminotransferase, ASM appendicular skeletal muscle mass, ASMI appendicular skeletal muscle index, AST aspartate aminotransferase, $B M I$ body mass index, CC calf circumference, COPD chronic obstructive pulmonary disease, CRP C-reactive protein, GGT $Y$-glutamyl transpeptidase, $H D L-C$ high-density lipoprotein cholesterol, $L D L-C$ low-density lipoprotein cholesterol 
Table 2 Group difference of the inflammatory indexes according to sarcopenia defined by different criteria

\begin{tabular}{|c|c|c|c|}
\hline Characteristics & No sarcopenia & Sarcopenia & $p$ \\
\hline AWGS & $n=323$ & $n=61$ & \\
\hline PLR & $94.3 \pm 39.5$ & $101.6 \pm 40.6$ & 0.190 \\
\hline NLR & $2.1 \pm 0.9$ & $2.1 \pm 0.8$ & 0.828 \\
\hline LMR & $4.4 \pm 1.3$ & $4.4 \pm 1.5$ & 0.939 \\
\hline AWGS 2019 & $n=208$ & $n=176$ & \\
\hline PLR & $93.7 \pm 35.5$ & $97.5 \pm 44.2$ & 0.348 \\
\hline NLR & $2.1 \pm 0.8$ & $2.1 \pm 1.0$ & 0.407 \\
\hline LMR & $4.4 \pm 1.5$ & $4.4 \pm 1.6$ & 0.757 \\
\hline EWGSOP & $n=339$ & $n=45$ & \\
\hline PLR & $94.2 \pm 39.6$ & $105.3 \pm 39.4$ & 0.079 \\
\hline NLR & $2.1 \pm 0.9$ & $2.3 \pm 1.2$ & 0.078 \\
\hline LMR & $4.5 \pm 1.5$ & $4.1 \pm 1.5$ & 0.141 \\
\hline EWGSOP2 & $n=346$ & $n=38$ & \\
\hline PLR & $94.5 \pm 39.7$ & $104.3 \pm 38.9$ & 0.149 \\
\hline NLR & $2.1 \pm 0.9$ & $2.3 \pm 1.1$ & 0.177 \\
\hline LMR & $4.4 \pm 1.5$ & $4.4 \pm 1.6$ & 0.909 \\
\hline IWGS & $n=288$ & $n=96$ & \\
\hline PLR & $94.1 \pm 38.6$ & $99.7 \pm 42.9$ & 0.228 \\
\hline NLR & $2.1 \pm 0.9$ & $2.1 \pm 0.9$ & 0.758 \\
\hline LMR & $4.4 \pm 1.5$ & $4.4 \pm 1.5$ & 0.884 \\
\hline FNIH & $n=325$ & $n=59$ & \\
\hline PLR & $96.1 \pm 40.2$ & $92.1 \pm 37.1$ & 0.484 \\
\hline NLR & $2.1 \pm 0.9$ & $1.9 \pm 0.8$ & 0.178 \\
\hline LMR & $4.4 \pm 1.5$ & $4.7 \pm 1.4$ & 0.078 \\
\hline
\end{tabular}

Abbreviations: AWGS Asia Working Group for Sarcopenia, AWGS 2019 the updated version of AWGS, EWGSOP European Working Group on Sarcopenia in Older People, EWGSOP2 the updated version of EWGSOP, FNIH the Foundation for the National Institutes of Health, IWGS International Working Group on Sarcopenia, $L M R$ lymphocyte-to-monocyte ratio, NLR neutrophil-to-lymphocyte ratio, PLR platelet-to-lymphocyte ratio

Moreover, similar results were found when sarcopenia was defined by the AWGS 2019, the EWGSOP, the EWGSOP2, the IWGS, or the FNIH, respectively (Table 4), and similar results were found when PLR, NLR, LMR, CRP were treated as categorical variables (using quartile cutoff points) regardless of the diagnostic criteria (Supplementary Table 3, 4, 5, 6, 7 and 8).

\section{Discussion}

This study showed that none of the three inflammatory indexes (PLR, NLR, or LMR) were significantly associated with sarcopenia in our study population regardless of the diagnostic criteria of sarcopenia. Additionally, no significant correlation between the three inflammatory indexes (PLR, NLR, or LMR) and serum CRP levels was found. However, PLR and LMR were significantly but slightly correlated with ASM and handgrip strength, respectively.

Our finding that the inflammatory indexes were not correlated with CRP levels and not associated with sarcopenia was not in line with previous studies. For example, a cross-sectional study conducted in the outpatient clinic found that NLR values were positively correlated with other inflammatory markers, such as CRP; and a higher NLR level was independently associated with an increased risk of EWGSOP defined sarcopenia [14]. The discrepancy may be related to the different settings of studies. In another cross-sectional study enrolled 3671 community-dwelling older adults, the PLR values were also positively correlated with CRP, and the elevations in PLR values were positively associated with sarcopenia status [15]. However, the determination of sarcopenia status was only based on the bioelectrical impedance analysis equation of Janssen et al. [23], which cannot reflect other important components of sarcopenia, such as muscle strength and physical performance. Based on present evidence, whether the inflammatory indexes can represent the inflammation in sarcopenia or be used as indicators of sarcopenia remains controversial.

In addition, our finding that CRP levels were not significantly associated with sarcopenia in communitydwelling older people was not in line with previous studies. For example, pooling data from 16 studies showed that sarcopenia was associated with a higher level of CRP [8]. The discrepancy may result from the different characteristics of the participants, those who included in these studies were more likely to have a greater degree of inflammation-driven by the underlying diseases, such as ankylosing spondylitis [24], ulcerative colitis [25], chronic obstructive pulmonary disease [26], end-stage renal disease [27], and malignancy disease [28-30].

Interestingly, we found that PLR and LMR were significantly but slightly correlated with ASM and handgrip strength respectively in the same study population. This finding was similar to previous studies. For example, in a cross-sectional study conducted in community-dwelling older adults, the elevations in PLR values were negatively associated with ASMI [15]. Meanwhile, a strong negative correlation was identified between free fat mass and NLR levels in sarcopenia patients admitted to the outpatient clinics [14].

The inflammatory indexes have been widely studied in patients with malignant diseases [12] and neurodegenerative diseases [13]. Some studies demonstrated that the inflammatory indexes were associated with other inflammatory indicators, such as IL-6, CRP, and TNF- $\alpha$, and were useful for predicting disease progression, treatment response, and prognosis [12, 31]. These findings imply 
Table 3 Correlations between the inflammatory indexes and ASM, gait speed, handgrip strength, and CRP

\begin{tabular}{|c|c|c|c|c|c|c|c|c|}
\hline & ASM & & Gait sp & & Handg & & CRP & \\
\hline & $r$ & $P$ & $r$ & $p$ & $r$ & $p$ & $r$ & $p$ \\
\hline$\overline{P L R}$ & -0.103 & $0.044^{*}$ & -0.026 & 0.611 & -0.076 & 0.135 & 0.004 & 0.942 \\
\hline NLR & -0.018 & 0.726 & 0.052 & 0.308 & 0.014 & 0.787 & 0.029 & 0.572 \\
\hline LMR & -0.100 & 0.051 & -0.072 & 0.162 & -0.121 & $0.018^{*}$ & -0.050 & 0.330 \\
\hline
\end{tabular}

Notes: *Significant at 0.05 level

Abbreviations: ASM appendicular skeletal muscle mass, CRP C-reactive protein, LMR lymphocyte-to-monocyte ratio, NLR neutrophil-to-lymphocyte ratio, PLR platelet-to-lymphocyte ratio, $r$ Pearson's $r$

Table 4 Association between PLR, NLR, LMR, CRP (per 1-SD) and sarcopenia according to Logistic Regression Models adjusted for potential confounders

\begin{tabular}{|c|c|c|c|c|}
\hline & Unadjusted & Model 1 & Model 2 & Model 3 \\
\hline \multicolumn{5}{|c|}{ AWGS-defined sarcopenia } \\
\hline PLR (per 1-SD) & $1.19(0.92-1.53)$ & $1.16(0.88-1.53)$ & $1.19(0.91-1.58)$ & $1.09(0.82-1.45)$ \\
\hline NLR (per 1-SD) & $0.97(0.73-1.28)$ & $1.04(0.77-1.40)$ & $1.04(0.77-1.40)$ & $0.96(0.71-1.30)$ \\
\hline LMR (per 1-SD) & $0.99(0.75-1.30)$ & $0.97(0.72-1.32)$ & $0.96(0.70-1.31)$ & $1.01(0.74-1.38)$ \\
\hline CRP (per 1-SD) & $1.11(0.86-1.44)$ & $1.07(0.81-1.42)$ & $1.06(0.80-1.41)$ & $1.14(0.85-1.54)$ \\
\hline \multicolumn{5}{|c|}{ AWGS 2019-defined sarcopenia } \\
\hline PLR (per 1-SD) & $1.10(0.90-1.35)$ & $1.09(0.89-1.35)$ & $1.10(0.89-1.36)$ & $0.86(0.67-1.10)$ \\
\hline NLR (per 1-SD) & $1.09(0.89-1.33)$ & $1.11(0.90-1.37)$ & $1.10(0.89-1.36)$ & $0.91(0.71-1.17)$ \\
\hline LMR (per 1-SD) & $0.97(0.79-1.19)$ & $1.00(0.81-1.25)$ & $1.01(0.82-1.26)$ & $1.15(0.89-1.47)$ \\
\hline CRP (per 1-SD) & $0.91(0.75-1.12)$ & $0.89(0.71-1.10)$ & $0.89(0.71-1.10)$ & $1.10(0.86-1.41)$ \\
\hline \multicolumn{5}{|c|}{ EWGSOP-defined sarcopenia } \\
\hline PLR (per 1-SD) & $1.28(0.97-1.69)$ & $1.27(0.95-1.70)$ & $1.27(0.95-1.70)$ & $1.01(0.74-1.38)$ \\
\hline NLR (per 1-SD) & $1.28(0.97-1.68)$ & $1.36(1.02-1.81)$ & $1.37(1.02-1.82)$ & $1.14(0.83-1.56)$ \\
\hline LMR (per 1-SD) & $0.78(0.56-1.09)$ & $0.77(0.54-1.10)$ & $0.75(0.53-1.08)$ & $0.86(0.58-1.27)$ \\
\hline CRP (per 1-SD) & $0.81(0.56-1.18)$ & $0.17(0.75-1.13)$ & $0.74(0.49-1.11)$ & $0.93(0.61-1.43)$ \\
\hline \multicolumn{5}{|c|}{ EWGSOP2-defined sarcopenia } \\
\hline PLR (per 1-SD) & $1.25(0.92-1.68)$ & $1.24(0.91-1.69)$ & $1.23(0.89-1.69)$ & $0.99(0.71-1.38)$ \\
\hline NLR (per 1-SD) & $1.23(0.91-1.65)$ & $1.28(0.94-1.75)$ & $1.30(0.95-1.77)$ & $1.11(0.79-1.54)$ \\
\hline LMR (per 1-SD) & $1.02(0.73-1.42)$ & $1.07(0.75-1.53)$ & $1.05(0.73-1.51)$ & $1.26(0.84-1.87)$ \\
\hline CRP (per 1-SD) & $1.00(0.72-1.40)$ & $0.97(0.68-1.38)$ & $0.95(0.66-1.36)$ & $1.17(0.79-1.74)$ \\
\hline \multicolumn{5}{|c|}{ IWGS-defined sarcopenia } \\
\hline PLR (per 1-SD) & $1.15(0.92-1.43)$ & $1.14(0.90-1.44)$ & $1.13(0.89-1.44)$ & $1.04(0.81-1.33)$ \\
\hline NLR (per 1-SD) & $0.96(0.76-1.22)$ & $0.99(0.77-1.27)$ & $0.98(0.77-1.26)$ & $0.92(0.71-1.18)$ \\
\hline LMR (per 1-SD) & $0.98(0.78-1.24)$ & $1.02(0.79-1.32)$ & $1.02(0.79-1.32)$ & $1.07(0.83-1.39)$ \\
\hline CRP (per 1-SD) & $0.96(0.76-1.22)$ & $0.92(0.78-1.19)$ & $0.92(0.71-1.19)$ & $0.98(0.75-1.28)$ \\
\hline \multicolumn{5}{|c|}{ FNIH-defined sarcopenia } \\
\hline PLR (per 1-SD) & $0.90(0.67-1.21)$ & $0.85(0.62-1.17)$ & $0.85(0.61-1.17)$ & $0.88(0.63-1.23)$ \\
\hline NLR (per 1-SD) & $0.81(0.59-1.10)$ & $0.82(0.59-1.15)$ & $0.83(0.59-1.16)$ & $0.85(0.60-1.20)$ \\
\hline LMR (per 1-SD) & $1.27(0.97-1.66)$ & $1.42(1.04-1.93)$ & $1.40(1.03-1.91)$ & $1.39(1.01-1.90)$ \\
\hline CRP (per 1-SD) & $1.09(0.84-1.42)$ & $1.05(0.78-1.41)$ & $1.04(0.77-1.40)$ & $1.02(0.74-1.40)$ \\
\hline
\end{tabular}

Notes: Data are presented as odds ratios (95\% confidential intervals). PLR, NLR, MLR were treated as continuous variables (per 1-SD)

Model 1: adjusted for age and gender. Model 2: adjusted for age, gender, coronary heart disease, and cognitive impairment. Model 3: adjusted for age, gender, coronary heart disease, cognitive impairment, albumin, $\mathrm{HDL}-\mathrm{C}$, and BMI

Abbreviations: AWGS Asia Working Group for Sarcopenia, AWGS 2019 the updated version of AWGS, CRP C-reactive protein, EWGSOP European Working Group on Sarcopenia in Older People, EWGSOP2 the updated version of EWGSOP, FNIH the Foundation for the National Institutes of Health, IWGS International Working Group on Sarcopenia, LMR lymphocyte-to-monocyte ratio, NLR neutrophil-to-lymphocyte ratio, $P L R$ platelet-to-lymphocyte ratio, $S D$ standard deviation 
that the inflammatory indexes may reflect the degree of inflammation in these diseases. However, other studies found the opposite results. For example, a prospective study conducted in 317 patients with muscle-invasive bladder cancer suggested that NLR was neither a prognostic nor a predictive biomarker for overall survival [32]. One explanation for the discrepancy of these results may be the reporting and publication bias. For example, in a meta-analysis about the correlation of NLR with outcomes of urothelial carcinoma, publication bias could not be excluded by inverted funnel plots [12]. An analysis of 1915 publications on cancer prognostic markers found that almost all articles reported statistically significant results [33]. Under strong reporting bias, the statistical significance may lose the ability to distinguish the importance of prognostic or predictive markers [33].

Some intrinsic defects of the inflammatory indexes may also limit their utility as biomarkers of various diseases. First, the mechanisms of how the inflammatory indexes predict disease prognosis remain unknown. As reported by Seok-Jin Choi, a high NLR indicated short survival duration in patients with amyotrophic lateral sclerosis (ALS), but there was no significant correlation between the NLR and CRP levels [13]. They suggested that a high NLR in ALS may not be a consequence of systemic inflammation, but may be due to the specific immune modulations underlying the pathogenesis of ALS. Second, it is difficult to establish optimal cut-off values for the inflammatory indexes. The current cutoff values widely vary across studies $[34,35]$. For example, Gary et al. identified that a PLR of 150 could serve as a cut-off value in patients with limb ischemia caused by peripheral arterial occlusive disease [36]. Another study suggested that a PLR of 116.85 for identifying sarcopenia in operable gastric cancer patients [37]. Moreover, the reported cut-off values for NLR as a poor prognostic indicator in patients with solid tumors varied from 1.9 to 7.2 [34].

Our study has some limitations. First, this was a crosssectional study conducted at a single institution. Second, the sample size of our study is relatively small to draw a robust conclusion. Third, we did not adjust for some important possible confounders, such as activities of daily living and frailty. Last, the calculation of these inflammatory indexes was dependent on a single measurement, therefore the accuracy of results could be affected by potential laboratory measurement errors and biologic variabilities.

\section{Conclusions}

In Chinese community-dwelling older people, the inflammatory indexes (PLR, NLR, and LMR) were not significantly associated with sarcopenia, although PLR and LMR were significantly but slightly associated with some components of sarcopenia (ASM and handgrip strength), respectively. Our study did not support these inflammatory indexes as the biomarkers for sarcopenia in Chinese community-dwelling older people. However, considering the inflammatory indexes can be simply calculated from a routine blood test without adding any burden to patients or medical staffs, further studies remain warranted to validate the association between the inflammatory indexes and sarcopenia in different populations, especially among those who are supposed to have low-grade chronic inflammation, such as the oldest old and the patients with diabetes, chronic obstructive pulmonary disease, or cancer.

\section{Supplementary Information}

The online version contains supplementary material available at https://doi. org/10.1186/s12877-020-01857-5.

Additional file $\mathbf{1}$ Table S1. The diagnostic criteria for sarcopenia used in this study.

Additional file $\mathbf{2}$ Table S2. Baseline characteristics of participants according to AWGS 2019-defined sarcopenia.

Additional file $\mathbf{3}$ Table $\mathbf{S 3}$. Association between PLR, NLR, LMR, CRP, and AWGS-defined sarcopenia according to Logistic Regression Models adjusted for potential confounders.

Additional file 4 Table S4. Association between PLR, NLR, LMR, CRP, and AWGS 2019-defined sarcopenia according to Logistic Regression Models adjusted for potential confounder.

Additional file 5 Table S5. Association between PLR, NLR, LMR, CRP, and EWGSOP-defined sarcopenia according to Logistic Regression Models adjusted for potential confounders.

Additional file 6 Table S6. Association between PLR, NLR, LMR, CRP, and EWGSOP2-defined sarcopenia according to Logistic Regression Models adjusted for potential confounders.

Additional file 7 Table S7. Association between PLR, NLR, LMR, CRP and IWGS-defined sarcopenia according to Logistic Regression Models adjusted for potential confounders.

Additional file 8 Table S8. Association between PLR, NLR, LMR, CRP, and FNIH-defined sarcopenia according to Logistic Regression Models adjusted for potential confounders.

\section{Abbreviations}

ALT: Alanine aminotransferase; ASM: Appendicular skeletal muscle mass; ASMI: Appendicular skeletal muscle mass index; AST: Aspartate aminotransferase; AWGS: Asian Working Group for Sarcopenia; AWGS 2019: The updated version of AWGS; BIA: Bioimpedance analysis; BMI: Body mass index; CC: Calf circumference; Cl: Confidence intervals; CRP: C-reactive protein; EWGSOP: European Working Group on Sarcopenia in Older People; EWGSOP2: The updated version of EWGSOP; FNIH: Foundation for the National Institutes of Health Sarcopenia Project; GGT: $\gamma$-glutamyl transpeptidase; HDL-C: High-density lipoprotein cholesterol; ICD-10CM: International classification of disease, tenth revision, clinical modification; IL-6: Interleukin- 6; IWGS: International Working Group on Sarcopenia; LMR: Lymphocyte-to-monocyte ratio; NLR: Neutrophil-to-lymphocyte ratio; OR: Odds ratio; PLR: Platelet-to-lymphocyte ratio; SD: Standard deviation; TNF-a: Tumor necrosis factor a 


\section{Authors' contributions}

Study concept and design: MY; acquisition of data: LX, LT, and XH; analysis and interpretation of data: MY, TT; drafting of the manuscript: $T$; critical revision of the manuscript: MY. All authors read and approved the final manuscript.

\section{Funding}

This study was funded by the National Natural Science Foundation of China (grant number 71704121) and the National Key R\&D Program of China (grant number 2018YFC2002104). The sponsor had no role in the design, methods, data collection, analysis, or preparation of the manuscript.

\section{Availability of data and materials}

Data that support the findings of this study are available from the corresponding author on reasonable request.

\section{Ethics approval and consent to participate}

The study protocol was approved by the Research Ethics Committee of Sichuan University. Written informed consent was obtained from all participants or their legal proxies.

\section{Consent for publication}

Not applicable.

\section{Competing interests}

The authors declare that they have no competing interests.

\section{Author details}

${ }^{1}$ Center of Gerontology and Geriatrics, West China Hospital, Sichuan University, No.37 Guoxue Lane, Chengdu, Sichuan, China. ${ }^{2}$ Precision Medicine Research Center, West China Hospital, Sichuan University, No. 37 Guoxue Lane, Chengdu, Sichuan, China. ${ }^{3}$ National Clinical Research Center for Geriatric Diseases at West China Hospital, Sichuan University, No. 37 Guoxue Lane, Chengdu, Sichuan, China.

Received: 22 July 2020 Accepted: 28 October 2020

Published online: 07 November 2020

\section{References}

1. Cruz-Jentoft AJ, Landi F, Topinkova E, Michel JP. Understanding sarcopenia as a geriatric syndrome. Curr Opinion Clin Nutri Metabol Care. 2010;13(1):1-7.

2. Cruz-Jentoft AJ, Bahat G, Bauer J, Boirie Y, Bruyère O, Cederholm T, Cooper C, Landi F, Rolland Y, Sayer AA, et al. Sarcopenia: revised European consensus on definition and diagnosis. Age Ageing. 2019;48(1):16-31.

3. Vellas B, Fielding RA, Bens C, Bernabei R, Cawthon PM, Cederholm T, CruzJentoft AJ, Del Signore S, Donahue S, Morley J, et al. Implications of ICD-10 for sarcopenia clinical practice and clinical trials: report by the international conference on frailty and sarcopenia research task force. J Frailty Aging. 2018;7(1):2-9.

4. Makizako H, Nakai Y, Tomioka K, Taniguchi Y. Prevalence of sarcopenia defined using the Asia working Group for Sarcopenia criteria in Japanese community-dwelling older adults: a systematic review and meta-analysis. Physical Therap Res. 2019;22(2):53-7.

5. Papadopoulou SK, Tsintavis P, Potsaki P, Papandreou D. Differences in the prevalence of sarcopenia in community-dwelling, nursing home and hospitalized individuals. A systematic review and meta-analysis. J Nutr Health Aging. 2020;24(1):83-90.

6. Meng SJ, Yu LJ. Oxidative stress, molecular inflammation and sarcopenia. Int J Mol Sci. 2010;11(4):1509-26.

7. Visser M, Pahor M, Taaffe DR, Goodpaster BH, Simonsick EM, Newman AB, Nevitt M, Harris TB. Relationship of interleukin- 6 and tumor necrosis factoralpha with muscle mass and muscle strength in elderly men and women: the health ABC study. J Gerontol A Biol Sci Med Sci. 2002;57(5):M326-32.

8. Bano G, Trevisan C, Carraro S, Solmi M, Luchini C, Stubbs B, Manzato E, Sergi $G$, Veronese N. Inflammation and sarcopenia: a systematic review and metaanalysis. Maturitas. 2017:96:10-5.

9. Luo H, Ge H, Cui Y, Zhang J, Fan R, Zheng A, Zheng X, Sun Y. Systemic inflammation biomarkers predict survival in patients of early stage nonsmall cell lung Cancer treated with stereotactic ablative radiotherapy - a single center experience. J Cancer. 2018;9(1):182-8.
10. Zahorec R. Ratio of neutrophil to lymphocyte counts--rapid and simple parameter of systemic inflammation and stress in critically ill. Bratisl Lek Listy. 2001;102(1):5-14.

11. Duffy BK, Gurm HS, Rajagopal V, Gupta R, Ellis SG, Bhatt DL. Usefulness of an elevated neutrophil to lymphocyte ratio in predicting long-term mortality after percutaneous coronary intervention. Am J Cardiol. 2006:97(7):993-6.

12. Suh J, Jung JH, Jeong CW, Kwak C, Kim HH, Ku JH. Clinical significance of pre-treated neutrophil-lymphocyte ratio in the Management of Urothelial Carcinoma: a systemic review and meta-analysis. Front Oncol. 2019;9:1365.

13. Choi S-J, Hong Y-H, Kim S-M, Shin J-Y, Suh YJ, Sung J-J. High neutrophil-tolymphocyte ratio predicts short survival duration in amyotrophic lateral sclerosis. Sci Rep. 2020;10(1):428.

14. Ozturk ZA, Kul S, Turkbeyler IH, Sayiner ZA, Abiyev A. Is increased neutrophil lymphocyte ratio remarking the inflammation in sarcopenia? Exp Gerontol. 2018;110:223-9.

15. Liaw FY, Huang CF, Chen WL, Wu LW, Peng TC, Chang YW, Kao TW. Higher platelet-to-lymphocyte ratio increased the risk of sarcopenia in the community-dwelling older adults. Sci Rep. 2017;7(1):16609.

16. Yang M, Hu X, Xie L, Zhang L, Zhou J, Lin J, Wang Y, Li Y, Han Z, Zhang D, et al. Screening Sarcopenia in Community-Dwelling Older Adults: SARC-F vs SARC-F Combined With Calf Circumference (SARC-CalF). J Am Med Dir Assoc. 2018;19(3):277.e271-8.

17. Chen LK, Liu LK, Woo J, Assantachai P, Auyeung TW, Bahyah KS, Chou MY, Chen LY, Hsu PS, Krairit O, et al. Sarcopenia in Asia: consensus report of the Asian working Group for Sarcopenia. J Am Med Dir Assoc. 2014;15(2):95-101.

18. Atkinson HH, Rosano C, Simonsick EM, Williamson JD, Davis C, Ambrosius WT, Rapp SR, Cesari M, Newman AB, Harris TB, et al. Cognitive function, gait speed decline, and comorbidities: the health, aging and body composition study. J Gerontol A Biol Sci Med Sci. 2007:62(8):844-50.

19. Chen LK, Woo J, Assantachai P, Auyeung TW, Chou MY, lijima K, Jang HC, Kang L, Kim M, Kim S, et al. Asian Working Group for Sarcopenia: 2019 Consensus Update on Sarcopenia Diagnosis and Treatment. J Am Med Directors Assoc. 2020;21(3):300-7 e302.

20. Cruz-Jentoft AJ, Baeyens JP, Bauer JM, Boirie Y, Cederholm T, Landi F, Martin FC, Michel JP, Rolland Y, Schneider SM, et al. Sarcopenia: European consensus on definition and diagnosis: report of the European working group on sarcopenia in older people. Age Ageing. 2010;39(4):412-23.

21. Fielding RA, Vellas B, Evans WJ, Bhasin S, Morley JE, Newman AB, Abellan van Kan G, Andrieu S, Bauer J, Breuille D, et al. Sarcopenia: an undiagnosed condition in older adults. Current consensus definition: prevalence, etiology, and consequences. International working group on sarcopenia. J Am Med Dir Assoc. 2011;12(4):249-56

22. Studenski SA, Peters KW, Alley DE, Cawthon PM, McLean RR, Harris TB, Ferrucci L, Guralnik JM, Fragala MS, Kenny AM, et al. The FNIH sarcopenia project: rationale, study description, conference recommendations, and final estimates. J Gerontol A Biol Sci Med Sci. 2014;69(5):547-58.

23. Janssen I, Heymsfield SB, Baumgartner RN, Ross R. Estimation of skeletal muscle mass by bioelectrical impedance analysis. J Appl Physiol (Bethesda, Md : 1985). 2000;89(2):465-71.

24. El Maghraoui A, Ebo'o FB, Sadni S, Majjad A, Hamza T, Mounach A. Is there a relation between pre-sarcopenia, sarcopenia, cachexia and osteoporosis in patients with ankylosing spondylitis? BMC Musculoskelet Disord. 2016;17:268.

25. Fujikawa H, Araki T, Okita Y, Kondo S, Kawamura M, Hiro J, Toiyama Y, Kobayashi M, Tanaka K, Inoue Y, et al. Impact of sarcopenia on surgical site infection after restorative proctocolectomy for ulcerative colitis. Surg Today. 2017:47(1):92-8

26. Joppa P, Tkacova R, Franssen FM, Hanson C, Rennard SI, Silverman EK, McDonald ML, Calverley PM, Tal-Singer R, Spruit MA, et al. Sarcopenic obesity, functional outcomes, and systemic inflammation in patients with chronic obstructive pulmonary disease. J Am Med Dir Assoc. 2016;17(8): 712-8.

27. Kim JK, Choi SR, Choi MJ, Kim SG, Lee YK, Noh JW, Kim HJ, Song YR. Prevalence of and factors associated with sarcopenia in elderly patients with end-stage renal disease. Clin Nutri. 2014;33(1):64-8.

28. Ishihara H, Kondo T, Omae K, Takagi T, lizuka J, Kobayashi H, Tanabe K. Sarcopenia and the modified Glasgow prognostic score are significant predictors of survival among patients with metastatic renal cell carcinoma who are receiving first-line Sunitinib treatment. Target Oncol. 2016;11(5): $605-17$. 
29. Kim EY, Kim YS, Seo JY, Park I, Ahn HK, Jeong YM, Kim JH, Kim N. The relationship between sarcopenia and systemic inflammatory response for Cancer Cachexia in small cell lung Cancer. PLoS One. 2016;11(8):e0161125.

30. Rollins KE, Tewari N, Ackner A, Awwad A, Madhusudan S, Macdonald IA, Fearon KC, Lobo DN. The impact of sarcopenia and myosteatosis on outcomes of unresectable pancreatic cancer or distal cholangiocarcinoma. Clin Nutri. 2016;35(5):1103-9.

31. D'Andrea D, Moschini M, Gust KM, Abufaraj M, Özsoy M, Mathieu R, Soria F, Briganti A, Rouprêt M, Karakiewicz Pl, et al. Lymphocyte-to-monocyte ratio and neutrophil-to-lymphocyte ratio as biomarkers for predicting lymph node metastasis and survival in patients treated with radical cystectomy. J Surg Oncol. 2017;115(4):455-61.

32. Ojerholm E, Smith A, Hwang W-T, Baumann BC, Tucker KN, Lerner SP, Mamtani R, Boursi B, Christodouleas JP. Neutrophil-to-lymphocyte ratio as a bladder cancer biomarker: assessing prognostic and predictive value in SWOG 8710. Cancer. 2017;123(5):794-801.

33. Kyzas PA, Denaxa-Kyza D, loannidis JP. Almost all articles on cancer prognostic markers report statistically significant results. Eur Cancer. 2007; 43(17):2559-79.

34. Templeton AJ, McNamara MG, Šeruga B, Vera-Badillo FE, Aneja P, Ocaña A, Leibowitz-Amit R, Sonpavde G, Knox JJ, Tran B, et al. Prognostic role of neutrophil-to-lymphocyte ratio in solid tumors: a systematic review and meta-analysis. J Nat Cancer Institute. 2014;106(6):dju124.

35. Dentali F, Nigro O, Squizzato A, Gianni M, Zuretti F, Grandi AM, Guasti L. Impact of neutrophils to lymphocytes ratio on major clinical outcomes in patients with acute coronary syndromes: a systematic review and metaanalysis of the literature. Int J Cardiol. 2018;266:31-7.

36. Gary T, Pichler M, Belaj K, Hafner F, Gerger A, Froehlich H, Eller P, Rief P, Hackl G, Pilger E, et al. Platelet-to-lymphocyte ratio: a novel marker for critical limb ischemia in peripheral arterial occlusive disease patients. PLoS One. 2013;8(7):e67688.

37. Lin J, Zhang W, Huang Y, Chen W, Wu R, Chen X, Lou N, Wang P. Sarcopenia is associated with the neutrophil/lymphocyte and platelet/ lymphocyte ratios in operable gastric cancer patients: a prospective study. Cancer Manag Res. 2018;10:4935-44.

\section{Publisher's Note}

Springer Nature remains neutral with regard to jurisdictional claims in published maps and institutional affiliations.

Ready to submit your research? Choose BMC and benefit from:

- fast, convenient online submission

- thorough peer review by experienced researchers in your field

- rapid publication on acceptance

- support for research data, including large and complex data types

- gold Open Access which fosters wider collaboration and increased citations

- maximum visibility for your research: over $100 \mathrm{M}$ website views per year

At $\mathrm{BMC}$, research is always in progress.

Learn more biomedcentral.com/submissions 